\title{
PREVENTIVE MEASURES IN NASAL SINUS THERAPY.*
}

\author{
By MYLES L. FORMBY, F.R.C.S. \\ (Asst. Surgeon, Ear, Nose and Throat Dept., University College Hospital; \\ Surgeon, Throat Nose and Ear Dept., Miller General Hospital.)
}

The exploration of the field of preventive medicine has during the past century been responsible for the abolition of most serious epidemics. In spite of the great advances that have taken place, the catarrhal infections of the upper respiratory tracts continue to thrive in no uncertain manner. The common cold, with its sequelæ, is as prevalent as ever, and our field of prevention presents an extremely barren corner when studied from the point of view of acute rhinitis and sinusitis. The seriousness of these conditions, as they exist at present, is exemplified in a paper by Gaudet, ${ }^{(3)}$ who points out that acute coryza and influenza are responsible for more lost time among industrial workers than all other diseases taken together. In conjunction with this statement I wish to stress the fact that these two common ailments are the two great precursors of sinus disease.

Although it is not possible for me to reveal to you anything new in the way of preventive measures as applied to the primary infection, I hope to describe therapeutic principles that are effective in the prevention and treatment of the usual complication, namely, sinusitis.

\section{Anatomy.}

A short experience as a demonstrator of rhinology has lead me to believe that students fail to grasp the pathology of sinus disease because they have no clear conception of the anatomical arrangement of the parts affected. The interior of the nose as depicted in the average text-book of anatomy consists of a long list of small structures with large names, which are huddled together in a confined inaccessible space between the two orbits. This space does not lend itself to dissection, and it is only with difficulty that the parts can be visualized in their normal relationship. Starting his clinical career with this initial disadvantage, the student is next invited to study the appearances of the diseased nose through the small anterior nares, and the even more unapproachable posterior choanæ. Associated with these difficulties is a feeling that the problem is most unlikely to arise in the Examination Hall and can therefore be safely dealt with after graduation. It is because I believe this to be a not unusual state of affairs, that I have chosen the question of nasal sinusitis for this lecture. If the subject matter is of a rather elementary nature, I hope you will forgive me.

In order to appreciate the structure of the nose and its accessory sinuses, detail must be avoided. There are two main compartments separated by the septum and each has four air spaces leading off from it. These are the sinuses-namely, maxillary, frontal, sphenoidal and ethmoidal. The first three are usually single spaces, while the ethmoid consists of a number of cells of varying size and shape, which fill up the corner between the other sinuses and the inner wall of the orbit. It is best likened to a piece of honeycomb, consisting of cells without uniformity.

The next important fact to grasp is that all these air spaces communicate with the main cavity of the nose. In the case of the three large spaces, this communication is through their respective ostia, while in the case of the "ethmoidal

* A Paper read before the Southampton Division of the British Medical Association, October 20 th, 1934. 
sinus," each cell has an ostium which opens either directly into the nose or into an adjacent cell. Though apparently a maze, there is intercommunication between all compartments in the healthy state.

The final anatomical point to bear in mind is that the lining of this enormous area is one continuous sheet of columnar ciliated epithelium, containing numbers of mucus-secreting cells. Only in the vestibule of the anterior naris and in the olfactory cleft is this type of mucous membrane not found.

Remembering this simple arrangement, it can be readily appreciated that wherever an infection begins in the nose, the pathological processes are the same. The ultimate morbid picture is determined by the nature and severity of the initial infection and the extent of its spread. In addition to this general conception of the process of nasal inflammation, it is well known that the maxillary sinus, owing to its proximity to the teeth, is not infrequently involved by infection that has spread directly from these structures. I do not propose to deal with the question of dental infection in sinus disease.

\section{Physiology.}

As a knowledge of the processes of health (as well of disease) in their application to the organs and parts concerned, is essential to intelligent treatment, before proceeding to a consideration of the infected nose, I will mention a few points in connection with the functions of the normal nose.

The main nasal cavities, in addition to sheltering the olfactory end organs, play an important part in the preparation of the inspired air for its entry into the lower respiratory passages. The function of the accessory sinuses, however, is not so definitely known. It is quite certain that they serve no function important to life. Embryologically they represent rudiments of the organs of smell, which in the lower animals reach a high degree of development. The suggestion that, man having assumed the upright posture, it became necessary to diminish the weight of the skull by converting compact bone into air cells, has little to support it.

It has now been definitely established that the sinuses take part in the preparation of the air on its way to the lungs. The actual amount of preparation for which the sinuses are responsible has not been determined. Most investigations have been made on the maxillary antra, but it can be safely assumed that the physiological principles are the same for all the air cells.

With inspiration warm air leaves the sinus and the pressure falls, while during expiration air enters the sinus and the pressure rises. With patent ostia the air pressure changes in the sinuses are identical with those in the nose. (Proetz.) ${ }^{(5)}$

The hypothesis that the air content of the cells influences the resonance of the voice receives strong support from some rhinologists, while others regard it as probably incidental. Tippins ${ }^{(8)}$ believes that with the sinuses lies our ability to communicate our ideas to one another by means of sound, either in speaking or singing. He points out that none of the lower animals living underground or in water have sinuses, while all animals that can produce any kind of sound have some form of sinus structure around their nasal cavities. The size and number of these cavities seems to correspond very closely to the range, carrying quality and 
volume of the sounds that they reflect. In man, who makes the most use of sound for communicating with his fellows, we find the sinuses most highly developed. In support of this theory, it is pointed out that the first indication that something is wrong with the sinuses is a change in the character of a person's speaking voice. Further, it is held that really good singers and speakers seldom have colds, while the common cold in its incipient stages can either be aborted or greatly alleviated by allowing sound vibrations a chance to set up similar vibrations in the sinuses.

Tippins considers the nasal accessory sinuses constitute the perfect " sounding board." The sound vibrations induced in and amplified by the sinuses bring about a natural massage of the lining mucous membrane, and this in turn ensures an adequate blood supply at the point in the body where the invading organisms of acute coryza first have a chance to gain a foothold. In his final paragraph the writer says, "I am forced to this astonishing, but nevertheless self proving, conclusion, that sinus infections are the direct result of abuses of the nose and throat by wrong habits of speaking and singing." While this statement is undoubtedly an exaggeration, I have quoted the theory at length because I believe it to have very definite practical value.

The researches of Proetz ${ }^{(6)}$ on the action of the cilia lining the nose and its sinuses have lead to discoveries of great importance. Proetz has shown that there is an orderly movement of cilia resulting in a propulsion of the mucus in each air cell towards its ostium, and in the nose towards the nasopharynx. Individual cilia bend slowly back and then quickly forward-a motion which Tilley ${ }^{(7)}$ compares to the perfect swing in golf-whipping the stream of mucus along in a constant direction. Normally this film of mucus is thin and of low viscosity; it travels towards the natural ostium at the rate of 5-1o mm. per minute, sweeping any foreign matter, such as organisms or particles of dust, along with it.

Now the presence of this thin film of mucus is essential for normal ciliary action, because as soon as an area of nasal mucosa is dried, the cilia in that area cease beating. By directly observing an area of mucous membrane while it is being dried, the cilia can be seen to become motionless as the dry area widens. If kept dry for I5-I8 minutes, it is necessary to flush the surface with normal saline for some time before activity is restored.

A state of affairs identical with that resulting from artificial drying can be produced in the nose and nasopharynx by interference with the normal air currents. A deflected nasal septum may result in a jet of air being projected on to one small area of mucosa and cause it to be constantly dry. Partial closure of the nasal chambers brought about by chilling, local irritation, intestinal upsets, or any other of the preliminary stages of an acute cold, produces a similar condition. By interfering with the proper ciliary propulsion of the mucus, the above abnormalities permit organisms to come to rest on the surface and so lay the membrane open to infection.

Thus we have a satisfactory explanation for the physio-pathological law propounded by Ballinger. (1) This law states that, "Obstruction of the drainage and ventilation of mucous membrane lined cavities predisposes them to infection and inflammation." I am of the opinion that this law expounds a profound truth and that the rational treatment of sinusitis must be based upon it. 


\section{Treatment.}

Rectifying Defects and Septic Foci.-It will be generally admitted in any discussion on therapeutics that the ideal to be aimed at is prevention. Applying this principle to the nose and bearing in mind the anatomical and physiological points mentioned above, it will be seen that the first essential is to have a nose as nearly normal as possible. Since the development of the nose depends upon that of the teeth and palate and since the sinuses are vestigial structures and therefore more prone to infection (c.f. the appendix), the chances of finding a perfectly normal organ would seem remote. In point of fact, few people have an ideal nose anatomically, but in spite of defects, the vast majority function quite satisfactorily.

Any gross deformity (e.g., a badly deflected septum) must be rectified. Equally, or even more important, is the treatment of any infection which may act as a constant source of local irritation. The usual sites for such infections are the teeth (either caries or pyorrhcea), the tonsils and-in children-the adenoids. It is futile to hope for success in the treatment of a nasal infection while chronic sepsis is lodged in any of these localities. A more difficult problem still is to deal with repeated infection from without. The community as a whole does not regard acute coryza and influenza or other forms of rhinorrhœa as conditions warranting isolation for part of their treatment. While this idea persists, epidemics will continue. Another copious mode of spread difficult to combat emanates from the family in which one of the members has a focus of infection-e.g., bad oral sepsis-and repeatedly infects the rest of the household. Rhinitis in children not infrequently arises in this manner.

The Common Cold.-Having dealt with the more obvious causes of recurrent nasal infections, how can one best treat the common cold in its incipient stage? The patient complains of slight nasal obstruction, a sense of discomfort and irritation in the nose-perhaps occasional sneezing; the nasopharynx feels dry and if left for 24 hours he knows he will develop a cold. My conception of the condition of the nose in such a case is that an area of mucosa-probably in one or more ethmoidal cells-is not draining adequately, so that the appropriate treatment is to ærate the affected area and provide the necessary drainage. I consider this is most satisfactorily accomplished by the method of "Displacement" described by Proetz. ${ }^{(4)}$

There are two factors which form the basis of this treatment:- The first is shrinkage of the mucous membrane and the second suction. The patient is placed flat on his back on a couch with his head extended over one end, so that the chin and external auditory meati are in the same vertical plane, and in such a way that fluid introduced at the anterior nares will run into the superior meatus and not down the naso-pharynx. This position is uncomfortable, and for the patient with a short neck difficult to assume. The desired position can also be obtained by fixing the patient to the couch and raising the foot until the body is at an angle of $45^{\circ}$ with the horizontal. With the head suitably placed, 4-5 c.c. of warm I\% Ephedrine in normal saline are run slowly into each nostril. Suction is now commenced, and this is best done with a Proetz syringe. With the syringe in one nostril, the other is closed by pressure with the finger, and while the patient says "cluck, cluck ..." in order to close off the nasopharynx below with the soft palate, intermittent suction is carried on for $30-40$ seconds. The syringe is then 
transferred to the opposite nostril and the process repeated. This is done two or three times on each side. The patient is then made to sit up at rest, allowing the excess of Ephedrine to run out of his nose or into his pharynx, but avoiding blowing the nose for as long as possible.

The effect of the Ephedrine is to shrink up the mucous membrane with which it comes in contact. It is used in preference to Adrenalin, because the latter paralyses ciliary action, while I\% Ephedrine does not. The effect of the suction is to create a partial vacuum in the nose and remove from the surrounding cells a small quantity of the air content. Now the solution of Ephedrine is lying over the ostia of these cells, and when the partial vacuum is released a few drops pass into the cells, causing a shrinking of the lining mucosa and allowing free aeration.

The number of cases so far treated does not permit me to state just how effective this method is in aborting a cold in the initial stage. As a means of preventing acute exacerbations in patients with a chronic catarrhal infection of the ethmoidal sinuses, it is highly successful in all cases. The treatment permits the sinuses to breathe, thus restoring normal function and aiding nature to combat infection. For this reason I regard it as an efficient preventive measure to be applied to all those cases of "stuffy" nose, which so frequently go on to acute rhinitis. Other important preventive measures are breathing exercises and singing. These are particularly applicable to children, since with them normal function is as essential for proper development as it is for averting the onset of disease.

Another preventive measure which is efficacious in some cases is inoculation, either with an autogenous or a stock vaccine. Patients should be carefully selected and a thorough examination made to exclude any cause for the repeated nasal infections before this treatment is tried. The natural resistance to any infection even in normal individuals becomes lowered from time to time, and if this resistance can be increased by inoculation at the appropriate moment, the treatment is ideal. Unfortunately, in the case of acute rhinitis, the exciting cause is unknown, so that success with a vaccine is a matter of chance. There is no doubt that patients suffering from chronic nasal infections, vasomotor rhinitis, or associated allergic conditions resulting from toxins supplied by organisms in the nose, derive great benefit from the use of an autogenous vaccine. In a large series of cases treated in this manner, $\operatorname{Cox}^{(2)}$ claims to have cured just over $70 \%$.

When an acute coryza has developed, an acute inflammatory process involves the mucosa of the nose and its sinuses. As with similar processes elsewhere in the body, this either resolves or advances to the condition of suppuration. The next step in treatment should be directed towards the prevention of this latter catastrophe.

The patient should be kept in bed during the "streaming" infectious stage and while there is any pyrexia. A saline purge should be given, the diet must be light and plenty of fluids taken. Aspirin and Dover's powder are the most suitable drugs to give, and quinine in the early stages does good in many cases. Local treatment consists of providing adequate ventilation and drainage of the nose and its cavities. With the mucosa in a state of acute hyperæmia, it must be irritated as little as possible and for this reason I consider any form of suction treatment bad. Inhalations of menthol, benzoin or eucalyptus are very efficacious 
and usually do all that is required. If the nasal airway is not cleared by these procedures, a weak solution of Ephedrine should be applied. This can be sprayed up as a $I \%$ aqueous solution in normal saline or run in the nostril as an oil-the proprietary preparation "Endrine" containing .75\% Ephedrine is very suitable. An adjunct to these familiar procedures is the application of infra-red light to the nose and sinuses. I have had no experience of this form of therapy, but it is strongly recommended by some authorities. (Gaudet.) ${ }^{(3)}$

If in the course of an acute catarrhal rhinitis which has been neglected, or one in which preventive measures have been unsuccessful, the patient's temperature rises (occasionally there is a rigor), the pulse rate increases, the nose becomes more obstructed, headache and neuralgic pains are complained of and the mucoid nasal secretion becomes purulent, then the inflammation has become suppurative. (I would here call attention to the fact that occasionally patients have severe pain and tenderness over one or more sinuses without suppuration being present. This most frequently occurs with the frontal sinus and is due to imperfect ventilation resulting from temporary blockage of the fronto-nasal duct.)

Suppurative Rhinitis.-In the early stages of "suppurative rhinitis" the treatment is expectant and a similar procedure to that detailed for the acute catarrhal stage is employed. It is now more important than ever that drainage should be free. To maintain an adequate airway more continuous therapy with more potent remedies must be employed. It is here that cocaine hydrochloride is of the greatest value. The most efficacious method is to moisten a pledget of cotton wool in a $5 \%$ Cocaine solution-I\% Ephedrine may be added with advantage-and to insert this high up into the middle meatus for Io-I5 minutes. This may be repeated four-hourly. Between the applications of Cocaine I advise giving inhalations, or a Chloretone Inhalant Spray, or Nasal Oil containing Menthol and Ung. Hydrarg. Ox. Flav. The oil, in addition to aiding drainage, protectso the excoriated mucous membrane and prevents unpleasant crusting about the? anterior nares. In patients who are susceptible to Cocaine, I use $2 \%$ Pantocaine. As an anæsthetic it is excellent, but the effect is of shorter duration than with Cocaine and the shrinkage produced is less. This latter defect can be overcome by adding Ephedrine.

During the acute stage of the suppurative process, so long as drainage is free, any form of lavage is unnecessary and it may be definitely harmful. Should, however, pus become shut off in a sinus, it must be evacuated by the simplest possible procedure. In most cases this can be done by the introduction of a cannula and aspiration of the pus or gentle lavage to the sinus. Where this procedure cannot be carried out, the appropriate intra-nasal operation for drainage must be done.

Finally, I should like to stress very forcibly that the treatment of acute conditions in the nose should be of the simplest possible nature. If preventive measures are employed at a sufficiently early stage, few infections will go on to chronic suppuration and the necessity for radical surgery will be almost completely obviated.

REFERENCES.

(1) Ballinger, W. L., Diseases of the Nose, Throat and Ear, 6th Ed., 1930, Henry Kimpton, p. 467.

(2) Cox, W. C., Military Surgeon, 1933, 73, 121-128.

(3) Gaudet, L. S., New Orleans, M. \& S. J., 1933, 86, 383-387.

(4) Proetz, Arthur W., Displacement, Annais Publishing Co., U.S.A., 1931

(5) Proetz, Arthur W., Annals of Otology, 1932, 41, 1117.

(6) Proetz, Arthur W., Annsls of Otology, 1933, 42, 778.

(7) Tilley, Herbert, Tr. Med. Soc. Lond., 1933, 56, 239-259.

(8) Tippins, E. E., Eye, Ear, Nose \& Throat Monthly, 1934, 12, 514-516. 